\title{
Noodle Soup: A Study into Nonbinary Movements
}

\author{
STEPHANIE SANG DELGADO
}

The Ohio State University

As digital interfaces become more prevalent in our daily interactions and conversations, a common misconception is that interfaces are only digital and therefore new. In reality, architecture has always been an interface. This paper, as well as the ACSA session that took place on September 14th, explores the possibilities of architectural interfaces. The following paper is broken into three main parts. The first is an explanation and description of Noodle Soup, the 2018 Ragdale Ring designed by office ca. The second part elaborates on how softness is used in other projects, referencing both architecture and art. The third part focuses on physics simulation as a design tool when using soft materials and the use of unconventional modelling methods to explore the relationship between static and dynamic forms

\section{NOODLE SOUP}

An interface is both a noun and verb. In its noun form, an interface is a point where two systems, subjects, or organizations meet and interact. As a verb, an interface means to connect or interact with. These definitions can be applied to the relationship users should be having with architecture. When interfaces are discussed, its digital form is emphasized. Instead the conversation should focus on both the digital and how built architecture can become an interface. If buildings become interfaces, designers can create new spaces that empower individual and collective agency. With this in mind, Noodle Soup, the 2018 Ragdale Ring designed by office ca, is an attempt to create an architectural scale interface.

Originally conceived as an interactive playscape, the installation is flexible enough for a range of outdoor performances, while being picturesque enough for it to seamlessly integrate into the wooded scenery of Lake Forest. To achieve the playscape, Noodle Soup uses two main elements: the walls and the noodles. The soft elements can interact with the hard structures to serve functional purposes such as seating, but they can also act as oversized toys, freely configurable in a variety of ways. The user can loop, knot, stack, and rearrange the noodles to shape the environment for their own needs. The walls and stage act as references and as constants for the ever-changing landscape.

The walls and stage are built from traditional wood framing and are the only fixed element in the composition. They were

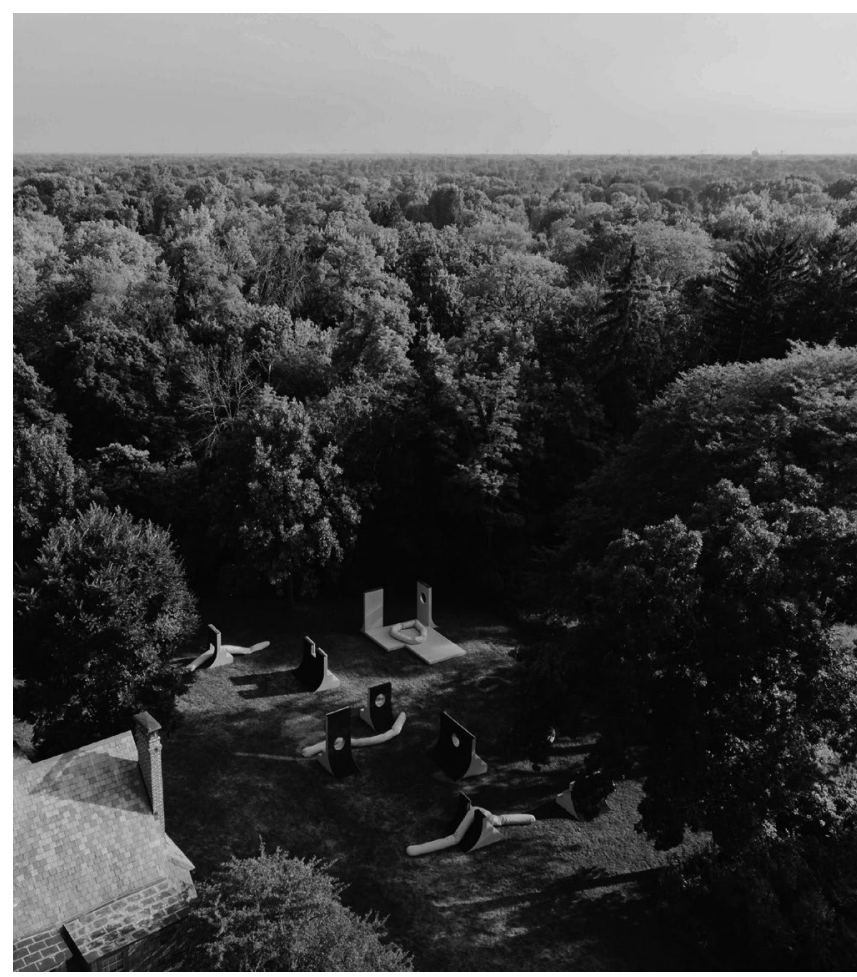

Figure 1: Aerial View of Noodle Soup

conceived as having been 'peeled' up from the ground on one side and sculpted into seating on the other. This contrast between natural and artificial is further articulated by having the concave side clad in a green shade of synthetic turf, blending it into the ground. The convex side is clad in a neon shade of turf so as to reinforce its artificial qualities. As the viewer makes their way around the composition, some walls recede into the greenery of the landscape, while others emerge to the foreground as geometric objects in a picturesque forest. View ports in the walls create both a visual connection to the other walls in the composition as well as window to the prairie and main house at the Ragdale residence.

The noodles are waterproof bean bags arranged throughout the composition in various lengths. They are made primarily of PVC coated polyester mesh fabric - which provides waterproofing for extended exterior use - filled with recycled foam peanuts. The fill is carefully customized so as to give the 


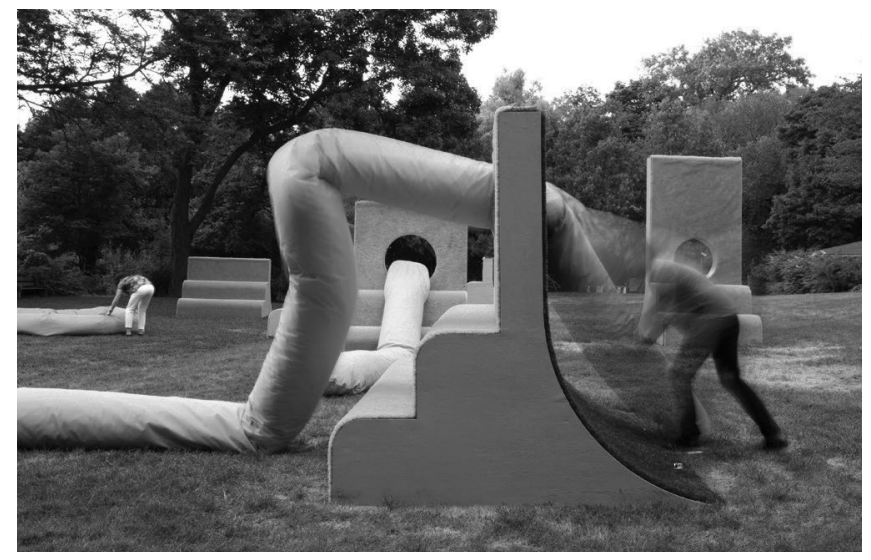

Figure 2: This photograph shows the noodles being rearranged throughout the composition.

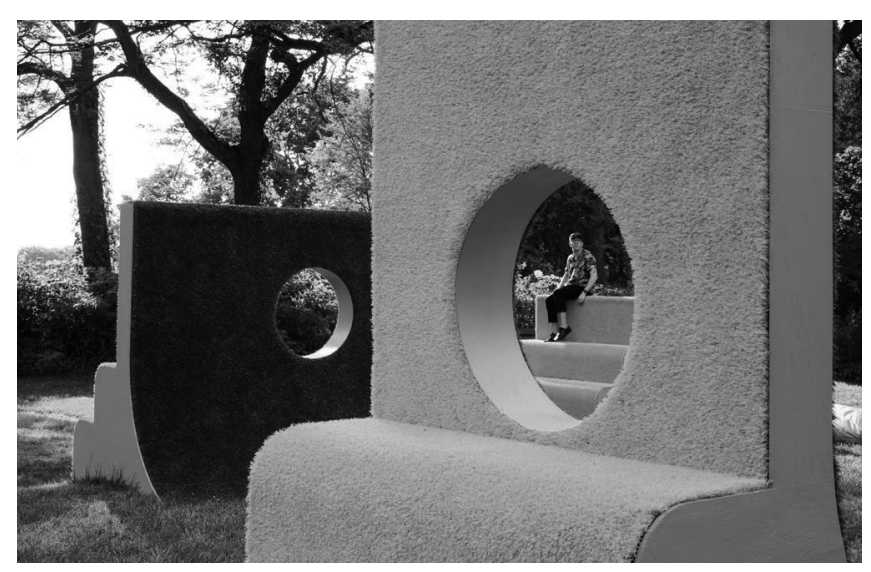

Figure 3: This photograph highlights the viewports in the walls.

noodle enough weight to withstand wind, but light enough so individual users can reposition them as they wish. Through this interface, Noodle Soup empowers the individual's artistic agency and blends whimsy, playfulness, and interaction into a transformable constructed landscape with both predictable and unpredictable results. By emphasizing the ever changing environment, the project entrusts the user with control over their surroundings.

\section{OTHER SOFT THINGS}

Designers are constantly creating user-specific spaces from the intimate act of designing a residential project to the specificity of departmental needs for a larger institutional building. However, designing a multi-user experience that serves all equally is not as commonplace or prevalent in our industry. By focusing on user agency, designs can be flexible in a new way. In Noodle Soup, user agency comes from the juxtaposition of the static elements with the freedom of the noodle-like bean bags. This method of using softness in architecture, allows for form and function to contrast between non-moving and moving elements.
In most buildings, the user has limited control of their environment. A user can open a window, close a door, or turn up the thermostat. These actions tend to be binary - open or shut, on or off, up or down. This is due to the rigid nature of building components. By focusing on softness, architecture can start to create non-binary movements and create more narratives. An example of everyday architectural softness is the curtain. Normally used for either stages or windows, the curtain amplifies the frame to focus a user's view to the stage or vista. In Maison Bordeaux ${ }^{1}$, the curtain plays a stronger role. They act as thresholds to highlight interior and exterior relationships instead of emphasizing the frame. In the exterior spaces of the house, the curtain is sheer providing a highly transparent border between the exterior sitting area and the outdoors. As you move through the house, the curtains' opacity increase as you move from public to private. In the living room, the white curtain is barely see-through. Its connection to the exterior becomes a hint and the curtain starts to act as wall with a circle window that is cut out of the curtain. This window can shrink, expand, and is constantly re framing the landscape. Lastly, the curtain in the bedroom is heavy, creating both a physical and visual barrier. The curtain's only indication of the exterior is through the sneak peek you get at the bottom as the curtain does not touch the ground.

But architectural softness can go beyond curtains. In the Humanoids by Ernesto Neto ${ }^{2}$, Neto creates both a collective and individual experience through soft, blobby forms that envelope the user into a never-ending hug. This hug encloses the user, acting like a sweater, the blob starts to become a room that blurs the boundary between the person and the enclosure. Humanoids examines how soft, and squishy objects can create new environments that can adjust to the scale of the user. It's almost as though there are various individual rooms within the actual the actual space. The project creates both a highly individual experience as well as a collective one.

In Mira Henry's Rough Coat installation, which was on exhibit at Sci-Arc in the Fall of 2018, explores architectural softness through a study on the monumental blanket. Usually understood as a cozy, comforting, and submissive, the installation focuses on re framing the blanket into a new monumental role that is normally reserved for stone and brick. The architectural softness in this project starts to act as a building envelope, hanging from a frame, and letting gravity take its toll. There are moments where the blanket falls neatly, and others where it doesn't. The exhibit becomes a memory of the performance, a freeze-frame to the ebb and flow of the fabric. Rough Coat showcases how softness can play a crucial role not only in the prioritizing user agency into a space but in creating cohabitations and overlapping narratives. 

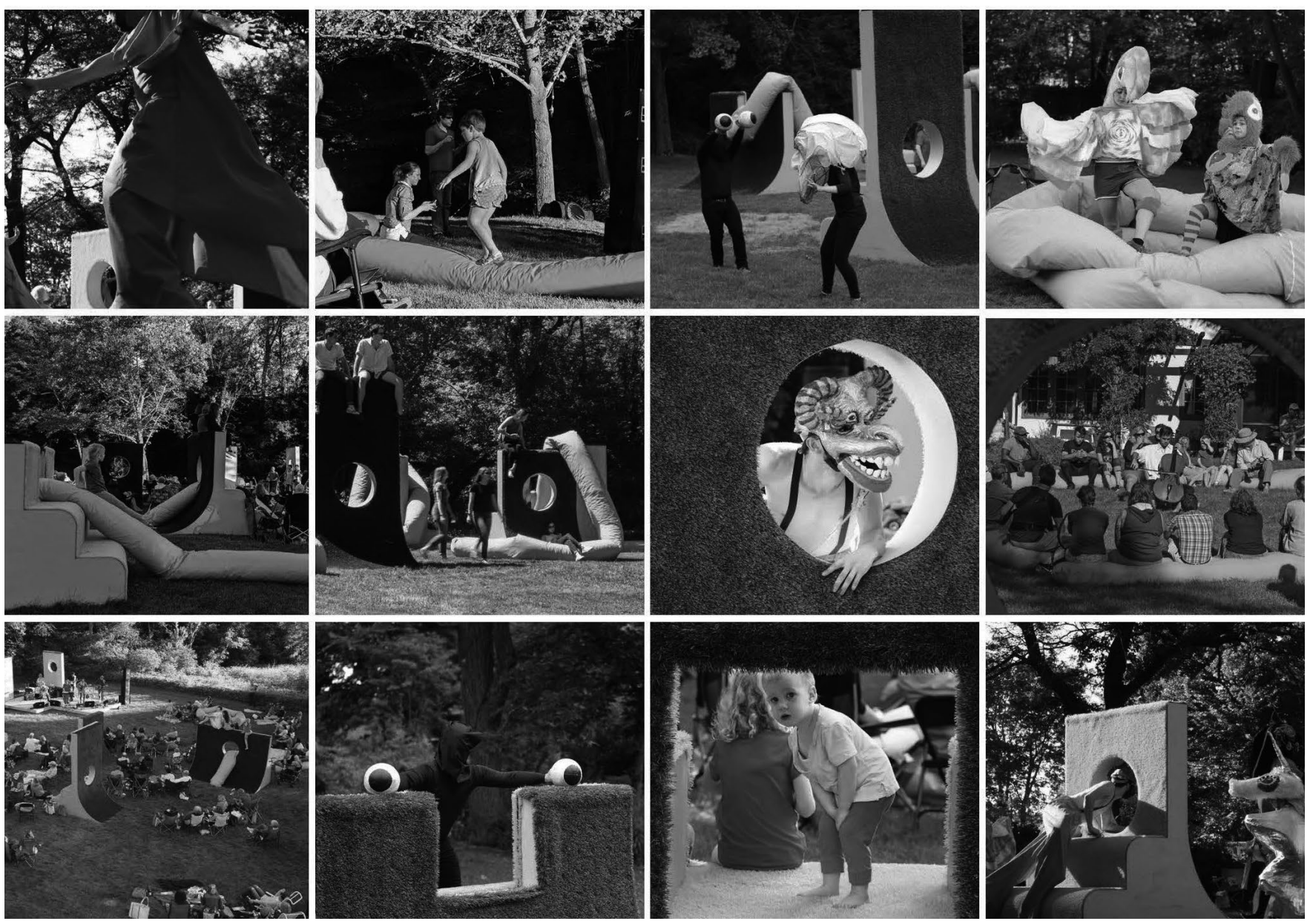

Figure 4: Compilation of different ways the project was used by users

\section{COOKED NOODLES AND DIGITAL NOODLES}

While questioning the role of softness in architectural design, we started to explore how softness can be an avenue for user interaction but also how its digital counterpart can give us an understanding how it will interact with other objects and, ultimately, with the users. For Noodle Soup, a web app called Ragdalenoodles.co was created to explore these possibilities. Through the web app, visitors could explore different configurations of the noodle-like bean bag and walls, but they could also draw their own. Through this web app, the users could almost test drive their interactions and find new ways to use the noodles. With digital physics simulations, designers can start to understand how materials will interact with each other. Programs such as 3Ds Max, allows for the designer to predict how dynamic materials can interact with static elements. Its digital presence allows for swift analysis on the performative nature of the design.

Even though digital modelling gives us an opportunity to explore these soft experiments in a controlled environment, using physical study models can help anticipate different ways that they will interact. To explore the possibilities of soft materials, looking into unconventional architectural modelling materials can be key in creating live-simulations of dynamic and static interactions. During the session, users were asked to explore how noodles, cooked and uncooked, can be used to create live-simulations. The users were provided with two types of noodles. Each of the noodles provide its own formal quality as well as its own limitations. The first was a Shan XI Pull Noodle, a thin, almost spongy, flat noodle that is strong enough to retain its shape but malleable enough to conform. The noodle can be dropped or draped over objects, its composition allows for it bend and flop but for the most part it retains its original form. The second noodle was not a noodle but instead was rice paper, a significantly thinner version of rice noodles. Rice paper come in the shape of discs and are hard and brittle. As the rice paper soaks up water, the paper changes to a soft and malleable material that molds itself unto any object that is draped on. As the rice paper dries and hardens, the form becomes fixed. The molded rice paper resembles that of a vacuum-formed object. Alongside the noodles, each user was given small blocks of wood and plexi. The users were tasked with using noodles and the blocks to create new compositions. 


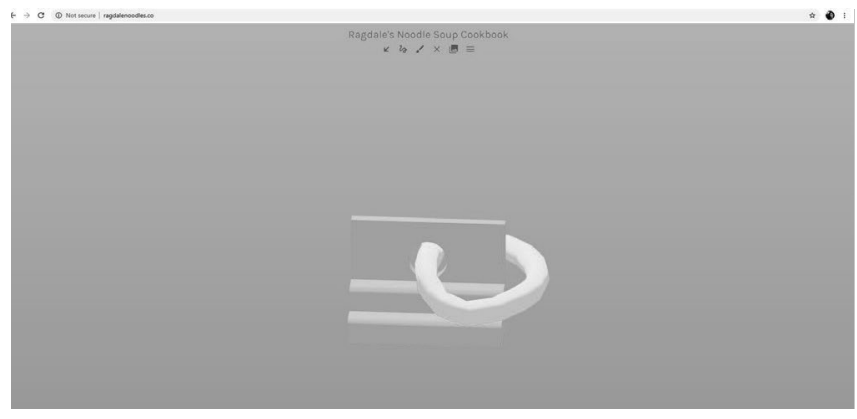

Figure 5: A screenshot of the Ragdalenoodles.co app

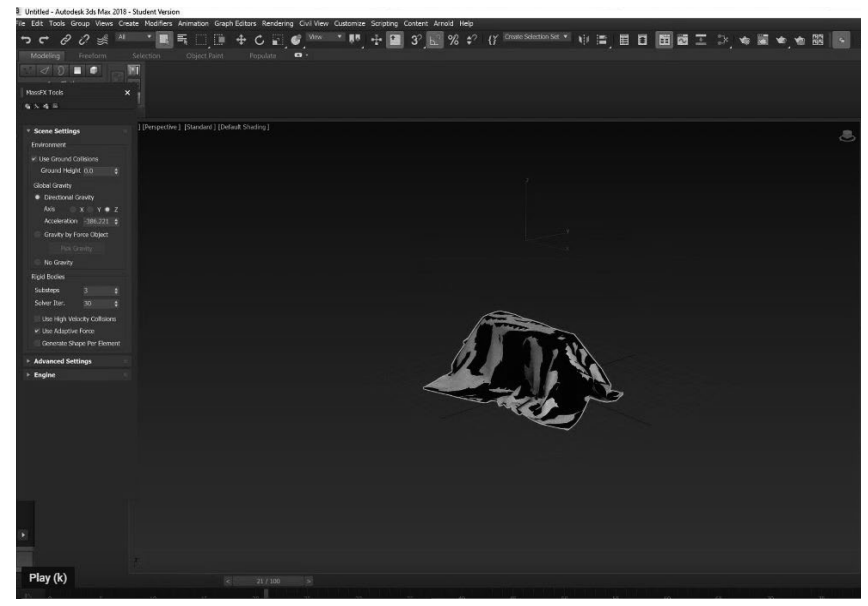

Figure 6: Screenshot of physics simulation using 3ds Max. The image shows the results of draping a fabric on top of a solid rectangle.

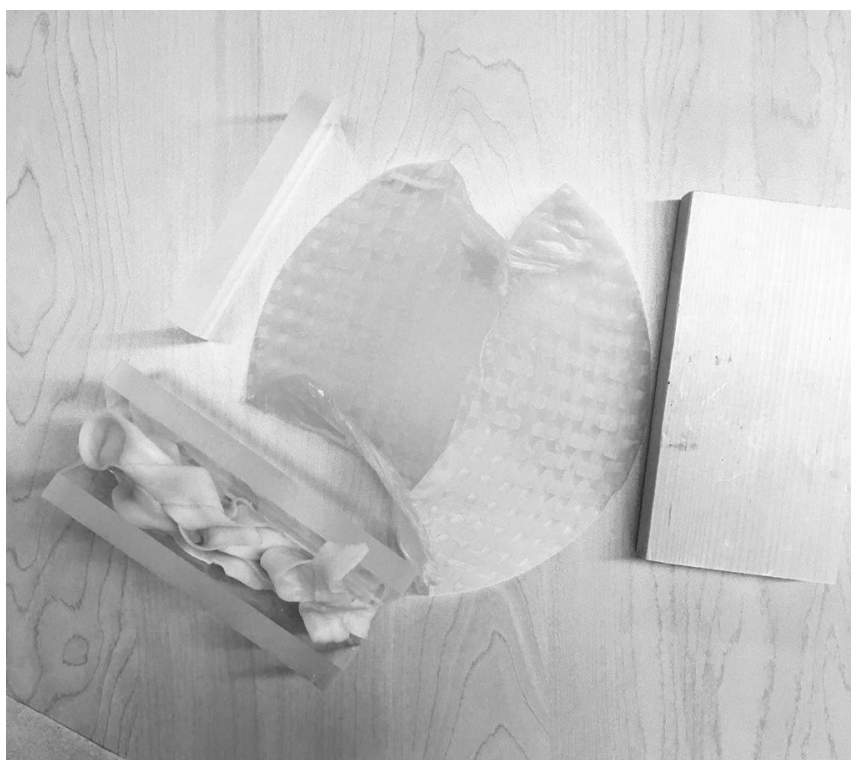

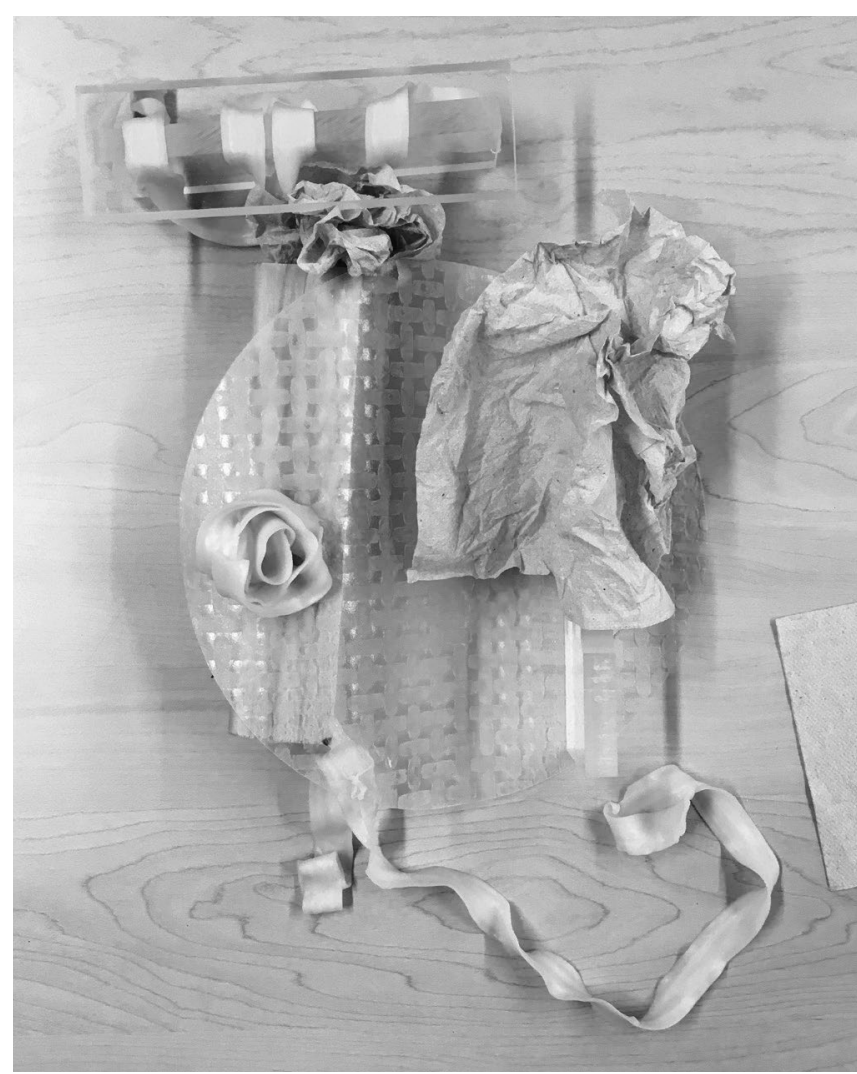

Figure 8: Workshop Results

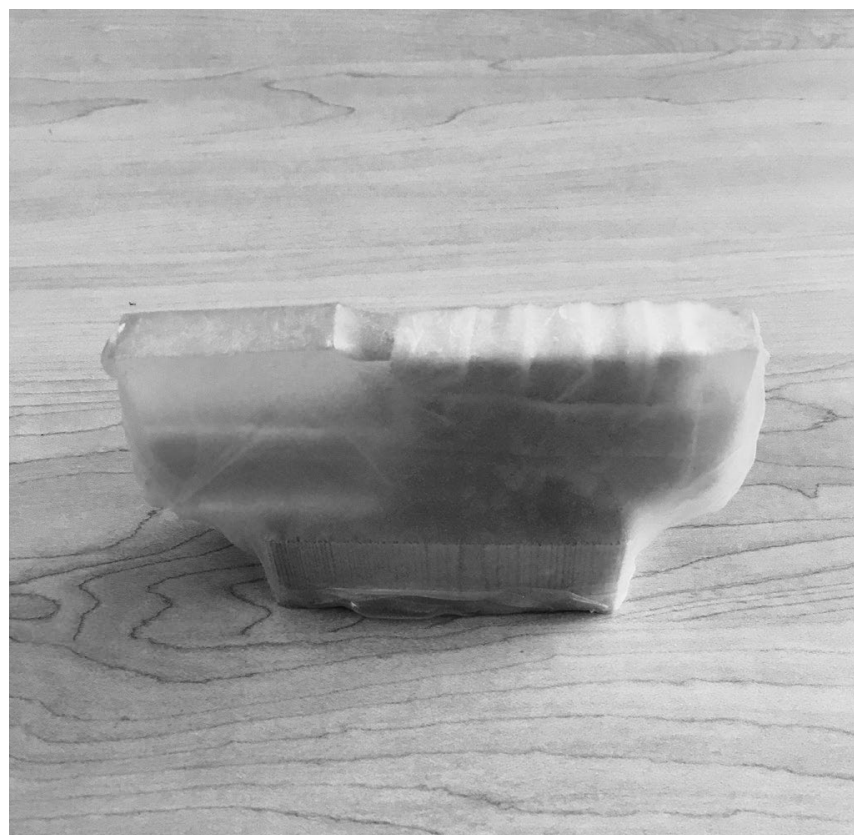

Figure 9: Workshop Results.

Figure 7: Workshop Results 
The activity asked the users to become playful, to reimagine how architectural form and function can be created. As seen below, the users created a series of configurations and combinations that exploited the nature of the materials. This exercise provided an opportunity to see how a common food, such as noodles, can be used for architectural design.

\section{CONCLUSION}

Why soft? When we look at buildings around us, we see that they are made of brick, steel, wood, concrete and other loadbearing materials. They become fixed to a specific time and place, never allowing itself to jump into a new era. Its fixed nature acts in opposition to the performative nature of users. By allowing architecture to become soft, we can allow buildings to join in the performance in ways that are more attuned with user actions.

\section{ENDNOTES}

1. "Maison a Bordeaux", OMA, September 1, 2019, https://oma.eu/projects/ maison-a-bordeaux

2. Neto, Ernesto, "Humanoids", Sculpture, 2014, Guggenheim Bilbao, https:// artdaily.cc/news/68238/-Ernesto-Neto--The-Body-that-Carries-Me--opensat-The-Guggenheim-Museum-Bilbao\#.Xctnp1dKhPY

3. Henry, Mira, "Rough Coat", Installation, 2018, Southern California Institute of Architecture, https://www.sciarc.edu/events/exhibitions/ mira-henry-rough-coat 